\title{
Norois
}

Environnement, aménagement, société

$203 \mid 2007 / 2$

Mobilité, hydrologie, loisir et tourisme, espace urbain

\section{De la station balnéaire à la ville littorale : trente ans de relations entre l'océan Atlantique et Pornic}

From seaside resort to coastal city: thirty years of relations between Atlantic ocean and Pornic

\section{Fabien Brulay}

\section{CpenEdition}

\section{Journals}

Édition électronique

URL : https://journals.openedition.org/norois/1517

DOI : $10.4000 /$ norois. 1517

ISBN : 978-2-7535-1551-2

ISSN : $1760-8546$

Éditeur

Presses universitaires de Rennes

Édition imprimée

Date de publication : 1 juin 2007

Pagination : 27-36

ISBN : 978-2-7535-0511-7

ISSN : 0029-182X

\section{Référence électronique}

Fabien Brulay, « De la station balnéaire à la ville littorale : trente ans de relations entre l'océan Atlantique et Pornic », Norois [En ligne], 203 | 2007/2, mis en ligne le 01 juin 2009, consulté le 13 janvier 2022. URL : http://journals.openedition.org/norois/1517 ; DOI : https://doi.org/10.4000/norois 1517 


\title{
DE LA STATION BALNÉAIRE À LA VILLE LITTORALE : Trente ans de relations entre l’océan Atlantique et Pornic
}

\author{
FABien Brulay \\ OTELO - JE 2433 \\ (Université de La Rochelle), \\ Faculté des Lettres, Langues, Arts et Sciences Humaines (FLLASH), \\ 1, parvis Fernand-Braudel - 17042 LA RocheLLE cedex \\ fabien.brulay@univ-lr.fr
}

\begin{abstract}
RÉSUMÉ
L'image construite par la municipalité de Pornic, longtemps station balnéaire familiale et populaire, est aujourd'hui celle d'une ville qui met en avant un cadre de vie fondé sur la proximité avec l'océan Atlantique. L'analyse des relations entre l'océan et le discours politique local, sur une période de trente ans, permet de mettre à jour les processus qui conduisent à cette évolution; ceux-ci sont indissociables des dynamiques territoriales qui prennent place sur la commune depuis quelques années (afflux de nouvelles populations, relations accrues avec Nantes et Saint-Nazaire, évolutions des pratiques touristiques).
\end{abstract}

Mots CLÉS : Aménagement - Géographie politique et sociale - Image - océan Atlantique - Représentations.

\section{ABSTRACT \\ From seaside resort to coastal city: thirty years of relations between Atlantic ocean and Pornic}

Since traditionnal sea resort along Atlantic french shoreline, the image of Pornic has become that of an Atlantic living environment. Over a period of thirty years, the study of relations between ocean and local policies discourse reveal the process of this development; those are also an integral part of current territorial dynamics (new populations settling, increased relationships with Nantes and Saint-Nazaire, new tourism frequentations)

KEY WORDS : Atlantic Ocean - Image - Local Planning - Political Geography-Representation.

Située sur le littoral océanique français (fig. 1), Pornic est une ville de 13639 habitants (recensement complémentaire de 2005) qui est passée du statut de port de pêche à celui de station balnéaire, bénéficiant des vertus thérapeutiques de l'eau de mer et, plus récemment, du développement du temps libre et des loisirs. De nos jours, Pornic constitue un centre urbain d'importance régionale qui profite des valeurs positives attribuées à l'océan Atlantique, comme l'évasion, la liberté, le naturel ou l'authenticité (Péron et Rieucau, 1996). L’océan est également perçu comme 


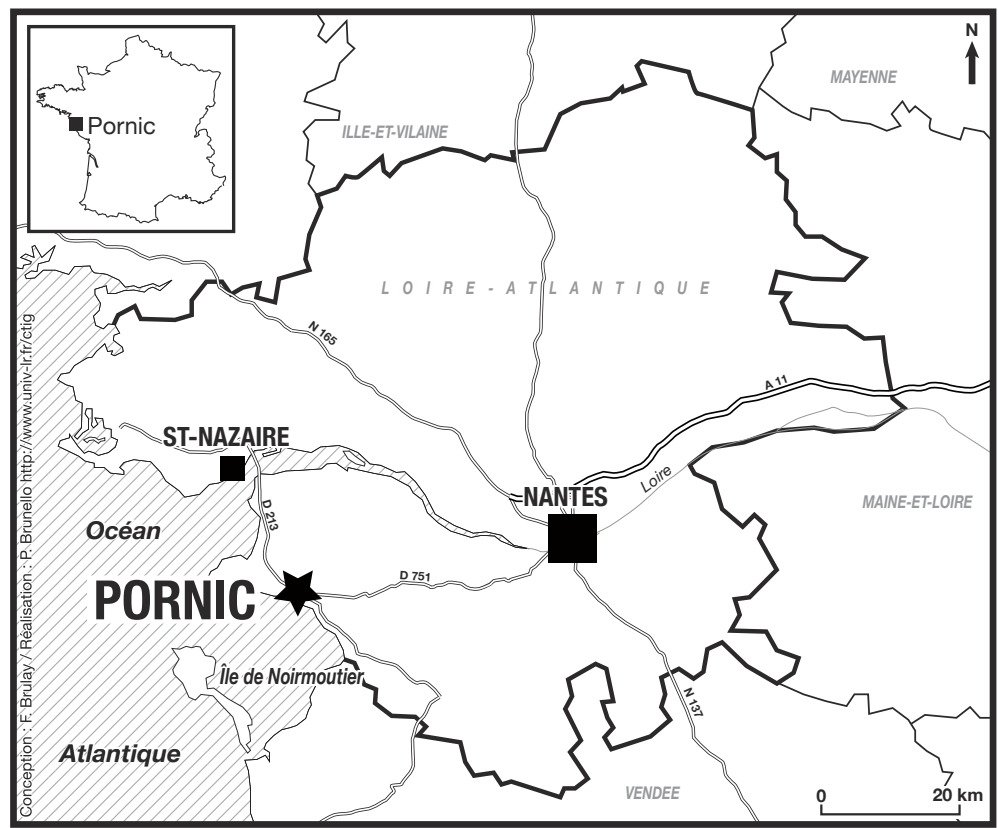

Figure 1 : Carte de localisation de Pornic

Pornic
Location Map of

le garant d'un cadre de vie agréable pour une société dominée par un mode de vie urbain, ce qui induit de fortes pressions sur les espaces littoraux (Datar, 2004). Cette perception contemporaine de l'élément océanique entraîne un renouvellement de sa mise en scène dans les villes littorales, par exemple dans les principaux ports français (Cabantous, Lespagnol et Péron, 2005). À Pornic les relations entretenues de nos jours avec l'océan Atlantique s'effectuent sur la base d'une diffusion plus large de l'environnement maritime dans l'espace urbain. Succédant à la forte influence du tourisme, cette diffusion donne naissance à un nouveau type d'urbanité.

Cette réflexion a pour objectif d'éclairer le passage de la cité balnéaire à celui de pôle urbain : l’importance accordée aux relations entre Pornic et l'océan dans l'image officielle construite par la municipalité constitue en ce sens un indicateur pertinent. Cela nécessite en premier lieu de mettre en lumière les ressorts de la communication politique locale, qui participe de l'action urbaine. L'analyse du contenu de ce discours, sur une période de trente ans, démontrera ensuite l'intégration croissante de l'environnement maritime dans les processus d'urbanisation de la ville; cette nouvelle relation à l'océan, indissociable d'enjeux territoriaux à l'échelle régionale, témoignant au final de l'avènement d'une urbanité post-touristique dans laquelle les préoccupations des résidents permanents importent plus que le développement des atouts balnéaires.

\section{Les enjeux de l'image de Pornic}

\section{L'IMAGE OFFICIELLE : UNE PRODUCTION DES SERVICES DE LA VILLE}

L'image officielle prend naissance dans la communication produite par les services qui sont à la disposition du pouvoir politique local, dans le cas présent la municipalité de Pornic. Périodiques d'informations, documents de planification spatiale, dépliants sont parmi les supports qui comptent le plus dans l'élaboration et la diffusion de l'image officielle de la ville. Ce type de publications participe par conséquent du vaste univers de la communication politique locale, qui produit de nombreux discours sur la ville, par exemple lors des déclarations des membres de l'équipe municipale (inaugurations, entretiens ou interviews) ou des opérations d'aménagement urbain. 
Si la communication politique locale est hétérogène et parfois spontanée, l'image officielle est le fruit d'une action collective, réfléchie et concertée, ce qui lui confère une plus grande cohérence (Faur, 1999).

À l'instar de toute image territoriale, une image officielle s'adresse à deux types de destinataires. Elle véhicule tout d'abord des propos à caractère touristique et relatifs à la publicité d'un territoire (le marketing urbain). Elle se construit également à partir d'un discours à destination des résidents qui s'attache principalement à informer sur la vie locale. Cependant ces deux aspects se rejoignent dans le fait que leur contenu est toujours fondé sur des références à l'histoire ou à la géographie locale. En effet, l'absence de références territoriales engendrerait des risques majeurs pour les producteurs, particulièrement dans les messages à destination des résidents. Une équipe municipale qui n'assurerait pas la satisfaction de ses administrés mettrait en péril la pérennité de sa présence et de ses fonctions. Il s'agit pour les producteurs de prendre constamment le pouls de ceux à qui l'image officielle se destine, c'est-à-dire de se conformer à certaines aspirations ou valeurs locales, à tout le moins celles du plus grand nombre : la convocation de référents territoriaux en constitue un des moyens.

L'intégration de nouveaux arrivants dans la fabrication de l'image officielle n'est donc pas chose aisée. Le cas de Pornic est en ce sens remarquable puisque la ville a vu sa population augmenter de moitié entre 1990 et 2005, en accueillant des migrants aux profils assez variés, des retraités mais aussi de nombreuses familles. N'en concluons pas pour autant que son image officielle s'est radicalement transformée : l'appel au territoire est toujours d'actualité car il contribue à la cohérence sémantique de l'ensemble; surtout l'intégration de nouveaux résidents dans la communication politique locale est un processus lent, qui prend soin de ne pas rompre avec un ordre discursif établi précédemment (Lussault, 1993).

D'autre part, la mobilisation de références territoriales résulte également des intentions de l'équipe municipale, qui les adapte à ses desseins. Par exemple, l'analyse de l'image officielle de Tours a montré les nombreux glissements opérés par la communication municipale vis-à-vis de certains référents territoriaux, ces derniers étant mobilisés au gré des projets urbains des élus locaux, en appelant tantôt au site originel et à la relation au fleuve, au milieu et à ses caractères, au paysage urbain hérité de la période Moderne ou à l'idée de «carrefour tourangeau » (Lussault, 1993). L'image officielle ne doit donc pas être réduite à un simple instrument au service d'une réélection : elle participe de l'action urbaine initiée par le pouvoir politique local. La communication des services municipaux, particulièrement ceux qui sont en charge de l'urbanisme, du cadre de vie ou de la communication, opère ainsi auprès des habitants pour instaurer une nouvelle représentation de la ville, qui permet de légitimer les projets urbains à venir. C'est le cas des références territoriales mobilisées autour de certains grands projets qui, outre le fait qu'elles témoignent de la présence de groupes de pressions locaux, proposent une nouvelle forme d'urbanité aux citoyens (Rosemberg, 2000). Ce procédé, qui constitue une étape préalable mais néanmoins nécessaire à la réalisation des projets urbains, confère une nature programmatique à la communication municipale.

L'image officielle de Pornic résulte par conséquent de la mise en tension d'un gouvernement urbain (la municipalité et la communication de ses services), avec la société locale et l'appropriation de références territoriales (géographiques et historiques) : ainsi forgée, elle constitue un ensemble sémique structuré qui médiatise les rapports au monde des individus et des groupes à l'espace (Lussault, 1998). L'analyse des évolutions de cette image officielle repose sur deux types de corpus. En premier lieu, les périodiques d'informations municipales sur une période de trente ans, qui permettent de repérer les discontinuités majeures du récit politique local; puis deux documents de planification spatiale (le projet de ZAC de la ria, et le PADD), qui permettent de cerner de façon plus précise les fondements de l'image officielle contemporaine, et nous renseignent aussi sur son devenir. 


\section{UNE RHÉTORIQUE AU SERVICE DE SES PRODUCTEURS}

L'analyse des publications périodiques, menée de façon diachronique, a consisté en premier lieu à relever tout ce qui faisait référence aux relations, passées et présentes, entre la ville et l'océan. Elle a abouti à un classement thématique en quatre catégories : pêche, tourisme balnéaire/plaisance, milieux littoraux et patrimoine maritime (matériel comme immatériel). Puis l'étude a été complétée par l'analyse des modalités de communication qui relèvent soit de la présence de l'équipe municipale (principalement celle du maire), soit des autres thèmes mobilisés dans le périodique. Le croisement des trois types de données a alors permis de caractériser différentes périodes dans la communication municipale. La mise en perspective des résultats obtenus par ce croisement avec les évolutions territoriales (dynamisme démographique, évolution des pratiques touristiques et urbanisation de la commune) a constitué l'ultime étape de la démarche méthodologique.

Les quelque soixante-dix magazines analysés sont parus entre 1976 et 2006, la périodicité du Pornic magazine devenant trimestrielle à la fin des années 1980. La communication municipale s'est ensuite étoffée, avec plusieurs hors séries, un supplément annuel (à partir de 1995) et une lettre mensuelle en 2005. La présence de trente ans de magazines témoigne de l'importante évolution de leur présentation, tant sur le plan graphique (usage de la couleur, multiplication des photos et des schémas explicatifs), que sur celui de la ligne éditoriale. Plus moderne, passant au besoin de l'information pratique sur la vie locale à l'explication parfois complexe de projets urbains, le ton adopté révèle aisément la professionnalisation d'une communication politique locale placée sous la responsabilité du maire (Devisme, 2000). Ce double processus de développement et de modernisation de la communication politique locale, qui s'inscrit dans une dynamique nationale à partir des années 1980, doit également beaucoup à l'arrivée de Philippe Boënnec en 1993 à la tête de la municipalité.

Adjoint dans l'équipe municipale précédente, il a été vice-président UMP du conseil général de Loire-Atlantique avant d'être déchu de ses fonctions à l'arrivée d'une majorité socialiste en 2004. Le maire actuel a été également un des élus les plus visibles au moment du naufrage de l'Erika, Pornic ayant subi d'importants dommages. Cela a contribué à renforcer sa présence dans la ville ainsi que dans le magazine municipal, où il est photographié plusieurs fois par numéro. À l'intérieur de celui-ci, la figure du premier magistrat repose sur deux piliers complémentaires : d'une part il se pose en guide, celui dont les choix sont en mesure de conduire les habitants vers le bien-être général. Cette posture se traduit par exemple au travers de nombreuses photos qui le montrent à la barre d'un bateau ou l'index «pointé vers l'avenir »; en outre il a longtemps signé son éditorial « docteur P. Boënnec », jouant de la dimension rassurante accordée à cette profession. L'analyse des documents révèle d'autre part la symbiose qu'il affiche avec son territoire d'élection, à travers une communication qui part du postulat que la ville constitue une entité indivisible. Le destin de Pornic y est ainsi présenté comme indéfectiblement lié à celui de son premier magistrat. Cette position privilégiée, loin d'être exceptionnelle dans ce type de supports de communication, lui permet de légitimer en parallèle des ambitions territoriales pour sa ville et des ambitions politiques personnelles. Dès lors la communication municipale s'adapte progressivement à ses desseins, devenant plus pragmatique et utilisant avec force les termes « objectifs », « bilan » ou " moyens ». Les suppléments annuels établissent par exemple un bilan détaillé de l'année écoulée et programment les réalisations à venir. Cependant les fréquents reproches de l'opposition municipale sur des ambitions souvent perçues comme démesurées témoignent de certains excès inhérents à une telle posture. Par exemple le projet d'aménagement de la ria, dont le coût est évalué aux environs de 35 millions d'euros, est largement dépendant des investisseurs privés. En outre l'absence de réflexions à une échelle extracommunale (faiblesse du fonctionnement intercommunal; inertie autour de l'élaboration du Schéma de Cohérence Territoriale) ${ }^{1}$ semble classer Pornic dans la catégorie des nombreuses communes littorales peu enclines à partager leurs richesses.

1. Certaines informations sont issues de deux entretiens menés avec le maire et un membre de l'opposition. 
On assiste en résumé à la professionnalisation de la communication politique locale depuis 1993. La municipalité pornicaise semble avoir pris la pleine mesure de la puissance de cet outil et des enjeux qui en découlent. Elle peut alors convoquer un imaginaire atlantique qui s'éloigne progressivement de celui mobilisé par l'ancienne municipalité.

\section{Quelle image pour Pornic aujourd'hui ?}

\section{L'OCÉAN ATLANTIQUE : UN FONDEMENT DE L'IMAGE CONTEMPORAINE DE PORNIC?}

L'arrivée de Philippe Boënnec marque en effet une première rupture dans les thèmes d'un magazine longtemps dévolu à la promotion des atouts touristiques. Auparavant, la valorisation de l'ensemble des relations entre Pornic et l'océan constituait l'essentiel de l'argumentaire : les photos et les articles sur les carrelets, les plages, les héritages balnéaires (casino, bâtis et architectures) ou la nouvelle thalassothérapie témoignaient de l'influence de ceux qui vivaient de la ressource touristique. À partir du milieu des années 1990, le poids de l'océan décroît progressivement, la valorisation de la situation littorale faisant surtout écho à un besoin d'ancrage territorial. Il s'agit essentiellement de rappeler la particularité géographique de la ville, et ce type d'argumentaire traite alors d'égal à égal avec d'autres référents territoriaux comme ceux qui suggèrent l'appartenance de la ville au Pays de Retz ou à la Bretagne (dans sa dimension rurale). Par ailleurs, la mobilisation d'un argumentaire atlantique en faveur du tourisme s'appuie principalement sur la plaisance (vie du port et courses en mer). L'imaginaire atlantique convoqué se réduit, contrairement à ce qui se produit dans certaines stations concurrentes de Vendée ou du nord de l'estuaire de la Loire (Brulay, 2007).

La place dévolue au littoral pornicais dans le discours municipal se trouve être un excellent révélateur de l'évolution des relations entre la ville et l'océan. Dans un premier temps, sous la municipalité de Gilbert Pollono, l'espace littoral est mobilisé dans toutes ses dimensions. Puis, c'est le seul bord de mer qui est valorisé à partir du milieu des années 1990. Le sentier côtier (situé sur la partie rocheuse de la ville) devient alors l'atout naturel de la commune, un joyau de forte valeur environnementale; comme souvent dans ce cas, l'argumentaire repose exclusivement sur sa grande fragilité. La pollution de l'Erika va contribuer à renforcer sa mobilisation par la municipalité. Celle-ci jette l'opprobre sur les profanateurs ce qui, dans le même temps, contribue à ériger ce sentier au rang de symbole de la ville (Debarbieux, 1996). Paradoxalement plus le sentier littoral est valorisé, plus l'urbanisation du territoire progresse vers l'intérieur des terres. Naufrage de l'Erika et urbanisation diffuse contribuent en tout cas à augmenter la dimension idéalisée du sentier littoral dans le périodique : les nombreuses manifestations de gratitude de Philippe Boënne envers tous ceux qui ont permis au sentier de retrouver sa pureté témoignent largement de la valeur qui lui est attribuée (fig. 2).

Enfin, en 2004 un supplément à Pornic magazine annonce le programme de restauration du littoral pornicais, dont le périmètre s'étend maintenant à l'ensemble de la frange littorale de la commune : il englobe les espaces terrestres les plus proches (parkings, chemins, végétation). Définissant une zone d'interface, cette opération matérialise l'étape la plus récente de la relation entre l'océan et la ville. Les discussions actuelles au sujet de la reconquête piétonnière des quais et du port semblent d'ailleurs confirmer cette volonté de la municipalité d'élargir le périmètre du littoral de la commune. Ces glissements sémantiques accompagnent un changement de stratégie de la part de la municipalité, qui ambitionne de diffuser l'influence de l'environnement maritime jusqu'aux limites communales.

\section{AMÉNAGEMENT DE LA RIA ET STRATÉGIE POLITIQUE LOCALE}

Une seconde rupture se produit en effet à l'orée des années 2000, même si elle prend naissance dès 1997. Un hors-série intitulé Pornic, projet de ville littorale est distribué cette année aux habi- 


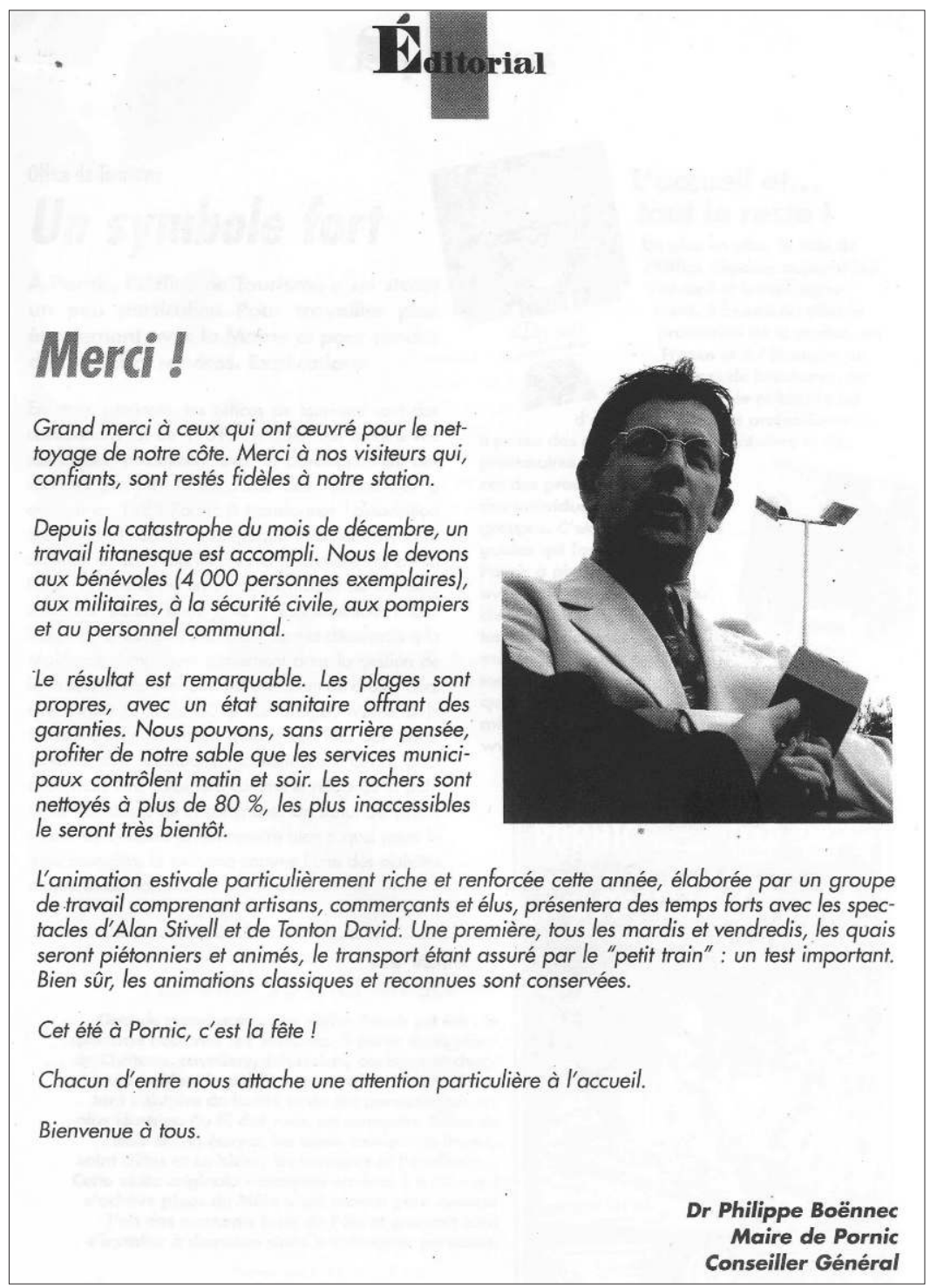

Figure 2 : Hommage et remerciement à ceux qui ont nettoyé le littoral pornicais dans l'éditorial du maire (Pornic Magazine, $\mathrm{n}^{\circ} 53$ - juillet 2000, p. 3)

gratitude to people Tribute
to people coastal zone of Pornic

tants et pose les bases d'une future «ville-centre ». Ce numéro spécial, qui fait état de relations privilégiées avec Nantes, suggère de faire de Pornic le pôle central de la rive gauche de l'estuaire de la Loire. L'accent est mis sur le fait que tous les secteurs d'activité de la ville y trouveront leur compte : saison touristique allongée, construction de nombreux équipements, développement des activités commerciales et artisanales sont parmi les principaux arguments avancés. Dès lors, les publications régulières, dont le ton s'est considérablement rajeuni, vont s'attacher à renforcer la dimension programmatique du projet de ville-centre, et les dossiers consacrés à la culture, à la famille, à l'éducation, à la proximité ou à la sécurité deviennent prédominants. Simultanément la posture rassurante du maire dans les magazines d'information municipale s'inscrit en parfait complément de ces transformations : elle annonce les changements à venir et, dans le même temps, tranquillise la population sur les capacités de l'équipe municipale à assurer la transition 
en douceur. En outre l'accès du maire au rang de conseiller général légitime les ambitions départementales.

Le numéro de l'été 2006 de Pornic magazine, dont la couverture titre « Un centre qui marque » symbolise l'avènement de cette stratégie locale : le centre-ville de Pornic est semble-t-il enfin adapté aux exigences d'un bassin de vie élargi, et la direction à suivre dans un futur proche est tracée. Le projet d'aménagement de la ria ${ }^{2}$, qui concourt à construire le récit politique local, confirme ce tournant. Il part du postulat qu'il existe trente-sept hectares délaissés (et pour la plupart non-urbanisés), visibles depuis la route Bleue. Jouxtant le centre-ville, cette zone constitue la porte d'entrée sud de Pornic : elle est destinée à tenir un rôle majeur dans la géographie locale. Il n'est alors pas étonnant de constater que ce projet est un concentré de l'image contemporaine de la ville : il tente de concilier les besoins de ceux qui désirent vivre à Pornic et de ceux qui vivent de l'économie locale.

Plus particulièrement, ce projet mobilise le registre sémantique que la localisation et les caractéristiques géographiques de la zone laissaient augurer : la philosophie du projet repose en effet sur la volonté d'assurer la continuité de la ria, la future ZAC constituant le prolongement de la partie maritime contemporaine (le port). La création d'un Parc de la Ria, dont les ambiances se déclineront en plusieurs entités aux toponymes atlantiques (îles, jardin des Marais), constitue une des réalisations majeures de l'opération; la présence abondante et fortement souhaitée de l'eau salée (par les remontées de marées) participe du même esprit. Le canal de Haute-Perche, au fond de cette vallée, constitue la ligne directrice de l'opération : en délimitant les versants, il distingue leurs vocations futures.

La zone située en amont de ce canal, sur la rive gauche (le parc de la Ria et, au-delà de la route Bleue, le val Saint-Martin), attend la construction de nombreux logements et offre de vastes espaces récréatifs; l'amélioration de son accessibilité (modernisation de la gare et de l'accès routier depuis la route Bleue) servant autant aux futurs résidents qu'à ceux qui viennent visiter la ville. Puis, au fur et à mesure que l'on s'approche de l'océan et du centre-ville, ces éléments de vie résidentielle diminuent au profit du renforcement du potentiel touristique de la commune : la rive droite, côté soleil, accueille en effet tous les équipements nécessaires à cette activité (hôtels et résidences de tourisme, équipements culturels et de loisirs). Et les nombreuses aires de stationnement prévues, qui libèrent les abords des espaces maritimes, contribuent également à accroître la valeur économique et symbolique du littoral pornicais. Par conséquent cette opération, qui veut contenir au-delà du canal les logements destinés aux résidents permanents, ambitionne dans le même temps d'augmenter l'attractivité touristique du centre-ville grâce à l'élargissement de son périmètre. Néanmoins, l'influence de l'océan dans la ville ne sera plus cantonnée au seul périmètre littoral, mais se propagera dorénavant beaucoup plus à l'intérieur des terres : l'imaginaire atlantique convoqué contribuera à rapprocher symboliquement les résidents permanents de l'océan, jouant sur le renforcement de la dimension idéalisée du littoral. La vie de la cité repose dorénavant sur une présence permanente de l'océan et de ses valeurs.

\section{VIVRE ENSEMBLE AU BORD DE L’OCÉAN}

Le Projet d'Aménagement et de Développement Durable (PADD), finalisé en 2005, s'inscrit logiquement dans le prolongement, mais à une autre échelle, de la stratégie de «ville-centre ». Intitulé «Vivre à Pornic en 2015 », ses orientations trahissent les mêmes volontés : premièrement multiplier les logements et les services sur le territoire communal, en prenant en compte les attentes des néo-résidents. La promotion d'arguments en faveur des espaces naturels (val SaintMartin) s'avère en ce sens d'un précieux secours. La volonté d'évoluer vers une activité touristique à la fréquentation moins importante, qui se place en rupture avec la station populaire des Trente Glorieuses, constitue le deuxième axe de cette vision du futur pornicais. Cette ambition se 
matérialise par la construction d'équipements nécessaires à la pérennité de l'activité dans la ville, particulièrement face à la concurrence féroce des autres stations du Centre-Ouest (Saint-Brévin, Les Sables-d'Olonne ou Saint-Jean-de-Monts notamment).

Ces deux éléments fondamentaux du PADD témoignent à nouveau du rôle essentiel assigné à l'océan : les acteurs politiques offrent la possibilité à tous ceux qui fréquentent Pornic, touristes comme résidents, d'y accéder et d'en profiter. Ici, à l'instar de nombreuses communes littorales, il est l'élément majeur dans la communication. Cependant les modalités de sa mobilisation sont particulières : si l'océan est rarement nommé, sa présence est par contre largement suggérée dans le projet. En fait, la valorisation de la situation littorale de la ville, de moins en moins récurrente dans la communication périodique, semble tellement évidente qu'elle disparaît du document de planification pour les quinze années à venir : elle y constitue une sorte de non-dit. La proximité avec l'océan s'inscrit par contre largement dans la réalité de la planification urbaine : elle est le fondement sur lequel les futurs aménagements viennent prendre place. Cette représentation latente de l'océan, qui accentue sa dimension idéelle, atteste également d'un cadre de vie qualitativement élevé : le PADD s'attache à rappeler aux habitants leur chance inestimable de pouvoir résider à Pornic, et la situation littorale y est présentée comme garante d'une vie urbaine harmonieuse pour les quinze ans à venir.

Cette stratégie de communication scinde la ville en deux parties, que l'on peut décrire comme le pendant métaphorique du modèle centre - périphérie : au cœur se trouverait la façade littorale et les lieux symboliques de la relation avec l'océan, comme le sentier mais aussi le port, les héritages des époques balnéaires. Au-delà se trouvent l'ensemble des attributs d'une ville et du mode de vie urbain, c'est-à-dire des logements, des emplois, des écoles et des relations avec les autres villes (Nantes et Saint-Nazaire principalement); d'ailleurs nombreux sont ceux qui suggèrent une intégration de Pornic à la métropole ligérienne (Davezies 2005, Fritsch, 2006). Ces espaces périphériques, qui débordent largement les limites communales, sont ici dévolus à la vie résidentielle : si la restauration de l'espace littoral permet aux habitants de ces espaces périphériques de se rendre auprès de l'océan, leur quotidien urbain en est en fait assez éloigné. Néanmoins, par le biais de la plus grande diffusion de l'environnement maritime, ils ont la possibilité de construire des relations symboliques avec l'élément océanique : celui-ci fait alors partie intégrante de leur vie quotidienne. Grâce au récit politique local, c'est l'ensemble de la ville qui se trouve sous l'influence de l'océan Atlantique (fig. 3). La communication municipale assure ainsi la liaison entre la réalité d'une vie urbaine (éloignement vis-à-vis de la bande côtière, mode de vie urbain et ses nécessités) et l'existence (imaginaire) de l'océan dans toute la ville. Elle s'évertue à réconcilier les aspirations et le mode de vie d'individus fondamentalement urbains (résidents comme touristes) à la recherche d'un cadre de vie littoral.

\section{Conclusion}

Depuis le milieu des années 1970, les relations entre l'océan Atlantique et les processus d'urbanisation de Pornic ont évolué en trois étapes successives. La première, orientée par le poids majeur de l'économie touristique, est de nature exclusivement balnéaire : l'interface littorale n'existe qu'au regard des équipements de la station. La seconde phase, celle de la transition, pose les bases du renouvellement des relations avec l'océan. Elle se fonde sur la valorisation d'un espace mythifié dans son entité, le bord de mer. Enfin l'ère «post-touristique », qui n'en est qu’à ses balbutiements, mise sur la promotion d'une nouvelle urbanité centrée sur une diffusion accrue de l'environnement maritime; elle trouve son origine dans les relations fortes avec la métropole ligérienne. 


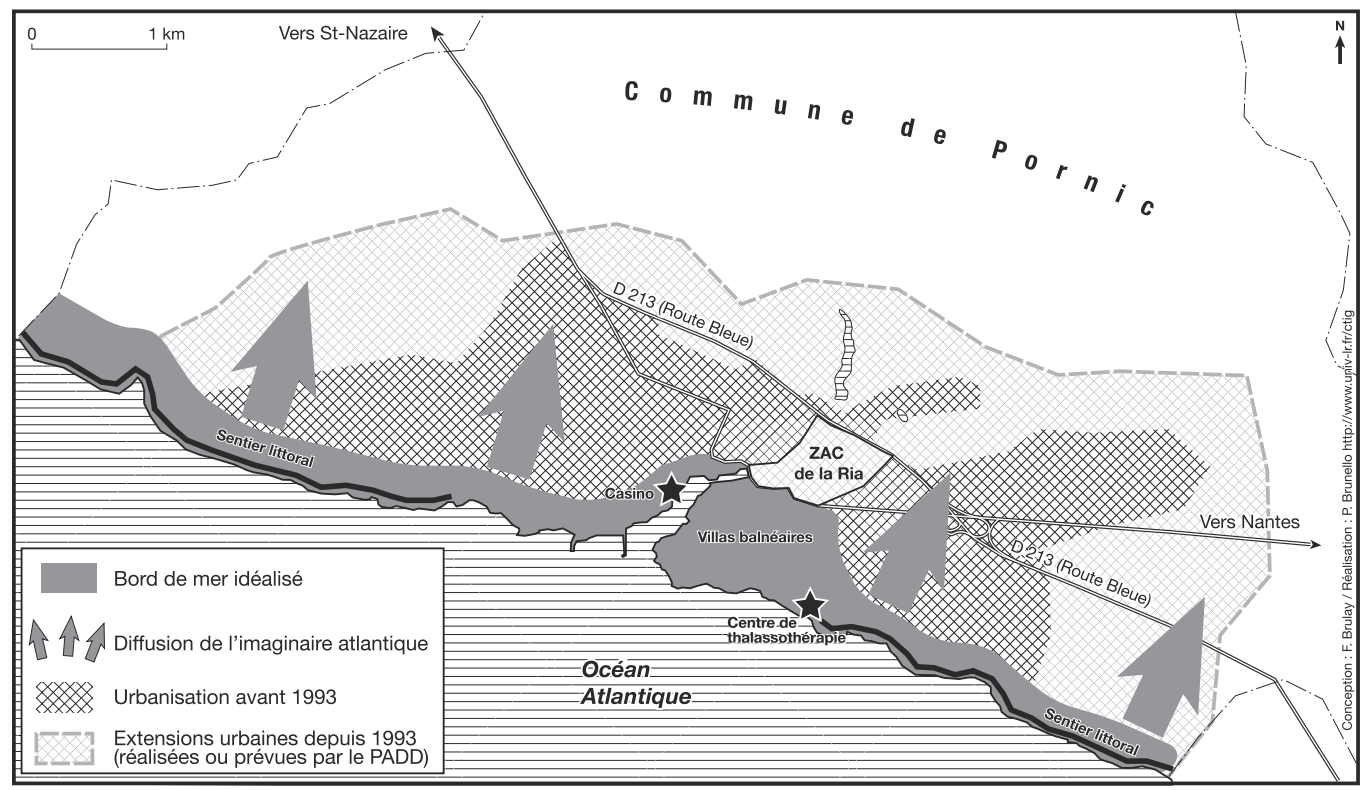

Figure 3 : L'océan Atlantique et la communication municipale de Pornic The Atlantic ocean and the municipal communication in Pornic

\section{Bibliographie}

Brulay (F), 2007. - «Océan et images urbaines de trois villes du littoral Atlantique (La Baule-Escoublac, les Sables-d'Olonne et Royan)», dans Viala (L), Villepontoux (S) (dir.), Imaginaires, Territoires, Sociétés : contribution à un déploiement transdisciplinaire de la géographie sociale, Montpellier, Presses Universitaires de Montpellier 3, coll. «Mutations des Territoires en Europe », p. 399-409.

Cabantous (A), Lespagnol (A), PÉron (F) (dir.), 2005. - Les Français, la terre et la mer XIX -XX $^{e}$ siècle, Paris, Fayard, 902 p.

DATAR, 2004. - Construire ensemble un développement équilibré du littoral, Paris, La Documentation Française, $156 \mathrm{p}$.

DaveZIES (L.), 2005. - Les moteurs du développement de la métropole Nantes-Saint-Nazaire, Nantes, rapport pour la Société d'Aménagement de la Métropole Ouest-Atlantique (SAMOA), 38 p.

Debarbieux (B.), 1996. - «Le lieu, fragment et symbole du territoire », Espaces et sociétés, n 82-83, p. $13-35$.

Devisme (L.), 2000. - « Identité urbaine et concurrence territoriale : la fabrique de l'image de Nantes », dans Carrière (J.-P.), FARThing (S.) (dir.), Les cités Atlantiques : villes périphériques ou métropoles de demain? diagnostics et politiques, Paris, Publisud, $356 \mathrm{p}$.

FAur (M.), 1999. - Bayonne et son image. Actions municipales et représentations 1983-1995, Bordeaux, CESURB, coll. « Recherches Urbaines », n¹3, 148 p.

Fritsch (B.), 2006. - « Nantes - Saint-Nazaire, métropole exemplaire? », L'information Géographique, $\mathrm{n}^{\circ} 4$, p. $25-45$.

Lussault (M.), 1993. - Tours : images de la ville et politique urbaine, Tours, Édition de la Maison des Sciences de la Ville, coll. «Science de la Ville », 416 p.

—, 1998. - «Images (de la ville) et politique (urbaine)», Revue de Géographie de Lyon, vol. 73, 1/98, p. $45-53$. 
Péron (F.), Rieucau (J.) (dir.), 1996. - La maritimité aujourd'hui, Paris, L'Harmattan, 335 p.

Rosemberg (M.), 2000. - Le marketing urbain en question : production d'espace et de discours dans quatre projets de villes, Paris, Economica, 184 p.

Cet article a été reçu le 23 mars 2007 et définitivement accepté le 19 juin 2007. 